\title{
Association Between 50 bp Insertion/Deletion Polymorphism in Promoter of the Superoxide Dismutase-1 and Temperament
}

\author{
Zahra Zendehboodi ${ }^{*}$ \\ 'Department of Biology, College of Sciences, Shiraz University, Shiraz, Iran
}

\author{
*Correspondence to \\ Zahra Zendehboodi, PhD; \\ Department of Biology, College of \\ Sciences, Shiraz University, Shiraz, \\ Iran. \\ Email: \\ zendehboodi@shirazu.ac.ir
}

Received March 1, 2018 Accepted March 15, 2018

Published online June 30, 2018

\begin{abstract}
Introduction: Temperament (mizaj) plays an important role in society's general health as well as in diagnosis and treatment of diseases. Despite its effectiveness, the mechanisms of temperament-dependent treatment in practical Iranian Traditional Medicine (ITM) has not been well understood yet. This study aimed to evaluate the association between the temperament and superoxide dismutase-1 (SOD1) polymorphism.

Methods: The study was carried out on healthy blood donors. All participants were from Fars province, southern Iran. To determine the participants' temperaments, a self-reported identification scale was used. Two hundred eighty males with any of 4 simple temperaments (Warm, Cold, Moist, and Dry) were included. The SOD1 genotypes were determined using a polymerase chain reaction (PCR)-based method. Multinomial logistic regression was used to calculate odds ratios (ORs) and $95 \% \mathrm{Cl}$ for the association of temperament with SOD1 polymorphism.

Results: No association was found between the insertion/deletion (Ins/Del) polymorphism of SOD1 and each of the temperament groups.

Conclusion: The results of this study indicated that the temperament is not affected by the Ins/ Del polymorphism of SOD1. Further research with larger samples and with other genes of antioxidant system is recommended.

Keywords: Temperament, Superoxide dismutase-1, Polymorphism
\end{abstract}

Please cite this article as follows: Zendehboodi Z. Association between 50 bp insertion/ deletion polymorphism in promoter of the superoxide dismutase-1 and temperament. Int J Basic Sci Med. 2018;3(2):59-

62. doi:10.15171/ ijbms.2018.11.

\section{Introduction}

Temperament (mizaj), one of the basic concepts of Iranian Traditional Medicine (ITM), plays an important role in maintaining the healthy state of an individual as well as in diagnosis and treatment of diseases. Temperament is believed to be a dominant quality resulting from the interaction of elements with different qualities (hot, cold, wet, dry) in the human body. In ITM, based on warmness and wetness intensity, the temperament is classified into 9 major groups: equilibrium group with equilibrium for both warmness and wetness; 4 simple groups (Warm, Cold, Moist, and Dry), each of them is in equilibrium for one quality and out of that for another quality; and 4 combined groups (Warm/ Moist, Warm/Dry, Cold/Moist, Cold/Dry), each of them is out of equilibrium for both
\end{abstract}

qualities. Healthy state is believed to be the result of the balanced temperament, and most of the diseases occur when this factor is disturbed. ${ }^{1,2}$

Variation in the temperament, which influences phenotypic traits such as morphological, physiological and psychological features, ${ }^{2}$ can be partially attributed to genetic differences. Considering the fact that genetic polymorphisms are involved in biodiversity, it is therefore of interest to explore the distribution pattern of genetic polymorphisms in different temperament modes. Definitely, identification of the genes related to this factor could help us better understand the molecular mechanism of ITM based treatments.

Superoxide dismutase-1 or SOD1 (EC 1.15.1.1; SOD1, OMIM: 147450) is an antioxidant enzyme localized in the cytosol, 
nucleus, peroxisomes, and mitochondria. It is a metaloenzyme which binds to copper and zinc and catalyzes the conversion of toxic superoxide radicals to hydrogen peroxide $\left(\mathrm{H}_{2} \mathrm{O}_{2}\right)$ and molecular oxygen $\left(\mathrm{O}_{2}\right){ }^{3}$ The loss of SOD1 activity, thus, could lead to the accumulation of reactive oxygen species (ROS). ROS, which play an important role in many physiological functions, are regularly produced in cells. ${ }^{4}$ However in excess, ROS may react with fatty acids, proteins and DNA, thereby causing damage or death to the cells. ${ }^{4}$

Several genetic polymorphisms of SOD1 have been shown to be associated with the risk of Alzheimer disease, liver and renal disease, cancer, and so on. ${ }^{5-10}$ There is a $50 \mathrm{bp}$ insertion/deletion (Ins/Del) polymorphism in the promoter region of this gene. The $50 \mathrm{bp}$-deleted region which binds to multiple transcription factors has been shown to be associated with reduced promoter and enzymatic activity of SOD $1 .{ }^{11-13}$

Previously, it was shown that the frequency of DNA damage (nuclear buds) and cell death biomarkers (condensed chromatin, karyorrhexis, pyknotic changes, and karyolitic cells) significantly differ between certain temperament groups. ${ }^{14}$ Moreover, the association of polymorphisms in antioxidant enzymes of glutathione S-transferase M1 and $\mathrm{T} 1$ and warm temperament has been reported. ${ }^{15}$ These findings support the idea that the antioxidant system may affect the temperament modes. Therefore, the present study was carried out to evaluate the association of temperament with SOD1 50 bp Ins/Del polymorphism as a potential genetic candidate.

\section{Materials and Methods}

The study was carried out on healthy individuals referred for blood donation to Shiraz Blood Transfusion Organization from November to December 2016. All participants were from Fars province, southern Iran, and aged between 20-40 years. To determine the participants' temperaments, a self-reported identification scale constructed for healthy individuals was used. ${ }^{16}$ Two hundred eighty males with equilibrium or any of 4 simple temperaments (warm, cold, moist, and dry) were included in the study. The primers and polymerase chain reaction (PCR) conditions for determining the SOD1 genotypes were the same as those reported previously. ${ }^{10}$ Multinomial logistic regression was used to calculate odds ratios (ORs) and 95\% CI for the association of temperament with SOD1 polymorphism.

\section{Results}

As revealed by the data of the age, no significant difference was observed between the means of age in different groups. The allele frequencies of SOD1 gene $50 \mathrm{bp}$ variant in each of the temperament groups is shown in Table 1. Because of low prevalence of the Del allele, Del/Del and Ins/Del genotypes were combined with each other as Del/- genotype for statistical comparisons. To control the possible effect of age, the association between SOD1 and temperament was examined using multinomial logistic regression while controlling for the age, and the equilibrium group was selected as the reference group. Neither the Ins/Ins nor the Del/- genotypes were associated with any of the simple groups (Table 2).

Table 1. Allele Frequencies of SOD1 Gene 50 bp Variant in Each Temperament Group

\begin{tabular}{lcccc}
\hline Temperament & Warm & Cold & Moist & Dry \\
\hline Allele Frequency $(\%)$ & & & & \\
Ins & $110(92)$ & $61(92)$ & $122(86)$ & $102(88)$ \\
Del & $10(8)$ & $5(8)$ & $20(14)$ & $102(88)$ \\
\hline
\end{tabular}

Table 2. Association Between Temperaments and SOD1 Polymorphism

\begin{tabular}{|c|c|c|c|c|c|c|c|}
\hline Temperament/Genotypes & $\mathbf{N}$ & Crude OR & $95 \% \mathrm{Cl}$ & $\boldsymbol{P}$ & Adjusted OR* & $95 \% \mathrm{Cl}$ & $\boldsymbol{P}$ \\
\hline \multicolumn{8}{|l|}{ Warm } \\
\hline Ins/Ins & 51 & 1 & & & 1 & & \\
\hline Del/- & 9 & 0.61 & $0.24-1.56$ & 0.304 & 0.63 & $0.24-1.61$ & 0.330 \\
\hline \multicolumn{8}{|l|}{ Cold } \\
\hline Ins/Ins & 28 & 1 & & & 1 & & \\
\hline Del/- & 5 & 0.62 & $0.20-1.92$ & 0.406 & 0.63 & $0.20-1.96$ & 0.426 \\
\hline \multicolumn{8}{|l|}{ Moist } \\
\hline Ins/Ins & 57 & 1 & & & 1 & & \\
\hline Del/- & 14 & 0.85 & $0.36-1.99$ & 0.708 & 0.89 & $0.38-2.11$ & 0.792 \\
\hline \multicolumn{8}{|l|}{ Dry } \\
\hline Ins/Ins & 46 & 1 & & & 1 & & \\
\hline Del/- & 12 & 0.90 & $0.37-2.19$ & 0.821 & 0.89 & $0.37-2.17$ & 0.801 \\
\hline
\end{tabular}

*Adjusted for age.

Reference group: Equilibrium (Ins/Ins = 45, Del/- = 13). 


\section{Discussion}

There are increasing evidence indicating the association of temperament with biological factors such as autonomic and immune systems, blood groups, enzymatic variation, and body composition. ${ }^{17-20}$ More interestingly, recent molecular biology studies have revealed that temperament modes are associated with distinct patterns of gene expressions as well as protein-protein interactions. ${ }^{21-23}$ The present results indicated that the temperament was not affected by the SOD1 Ins/Del polymorphism. This is the first study on the association of SOD1 polymorphism with temperament.

The sample size which might not be large enough to reach a convincing conclusion is the limitation of the present study. Another limitation of this study which should be taken into consideration is the unavailability of general health status of participants. However, despite the fact that physicians ignore some diseases during screening, it should be noticed that usually most of volunteers verified for blood donation are in well physical and mental health.

\section{Conclusion}

In conclusion, this study did not support the association between SOD1 Ins/Del polymorphism and any of the temperament modes. However, Ins/Del polymorphism of SOD1 needs to be further analyzed in future studies to verify our observation. Performing larger studies with greater samples and with other SOD1 polymorphisms as well as other polymorphisms in genes of antioxidant system is recommended.

\section{Ethical Approval}

This study was approved by Ethics Committee of Shiraz University (ECBDE-SU-9-6177616), and each subject was informed about the objectives of the study.

\section{Competing interests \\ Not declared.}

\section{Acknowledgements}

The author greatly thanks all the individuals who consented to participate in this study. This study was supported by Shiraz University.

\section{References}

1. Rezaeizadeh H, Alizadeh M, Naseri M, Shams Ardakani M. The Traditional Iranian Medicine point of view on health and disease. Iran J Public Health. 2009;38(Suppl 1):169172.

2. Yousefifard M, Parviz M, Hosseini M, Ebadiani M, Keshavarz M. Mizaj; past, present and future. Physiol Pharmacol. 2013;16(4):328-339.

3. Milani P, Gagliardi S, Cova E, Cereda C. SOD1 transcriptional and posttranscriptional regulation and its potential implications in ALS. Neurol Res Int. 2011;2011: 458427. doi:10.1155/2011/458427
4. Maes M, Galecki P, Chang YS, Berk M. A review on the oxidative and nitrosative stress (O\&NS) pathways in major depression and their possible contribution to the (neuro)degenerative processes in that illness. Prog Neuropsychopharmacol Biol Psychiatry. 2011;35(3):676692. doi:10.1016/j.pnpbp.2010.05.004

5. Kim SH, Kim SH, Lee JH, et al. Superoxide dismutase gene (SOD1, SOD2, and SOD3) polymorphisms and anti-tuberculosis drug-induced hepatitis. Allergy Asthma Immunol Res. 2015;7(1):88-91. doi:10.4168/ aair.2015.7.1.88

6. Spisak K, Klimkowicz-Mrowiec A, Pera J, Dziedzic T, Aleksandra G, Slowik A. rs2070424 of the SOD1 gene is associated with risk of Alzheimer's disease. Neurol Neurochir Pol. 2014;48(5):342-345. doi:10.1016/j. pjnns.2014.09.002

7. Ebrahimpour S, Saadat I. Association of CAT C-262 T and SOD1 A251G single nucleotide polymorphisms susceptible to gastric cancer. Mol Biol Res Commun. 2014;3(4):223229.

8. Neves AL, Mohammedi K, Emery N, et al. Allelic variations in superoxide dismutase-1 (SOD1) gene and renal and cardiovascular morbidity and mortality in type 2 diabetic subjects. Mol Genet Metabol. 2012;106(3):359-365. doi:10.1016/j.ymgme.2012.04.023

9. Mohammedi K, Maimaitiming S, Emery N, et al. Allelic variations in superoxide dismutase-1 (SOD1) gene are associated with increased risk of diabetic nephropathy in type 1 diabetic subjects. Mol Genet Metabol. 2011;104(4):654-660. doi:10.1016/j.ymgme.2011.08.033

10. Eskandari-Nasab E, Kharazi-Nejad E, Nakhaee A, et al. 50-bp Ins/Del polymorphism of SOD1 is associated with increased risk of cardiovascular disease. Acta Med Iran. 2014;52(8):591-595.

11. Broom WJ, Greenway M, Sadri-Vakili G, et al. 50 bp deletion in the promoter for superoxide dismutase 1 (SOD1) reduces SOD1 expression in vitro and may correlate with increased age of onset of sporadic amyotrophic lateral sclerosis. Amyotroph Lateral Scler. 2008;9(4):229-237. doi:10.1080/17482960802103107

12. Ingre C, Wuolikainen A, Marklund SL, Birve A, Press R, Andersen PM. A 50 bp deletion in the SOD1 promoter lowers enzyme expression but is not associated with ALS in Sweden. Amyotroph Lateral Scler Frontotemporal Degener. 2016;17(5-6):452-457. doi:10.3109/21678421.20 16.1159223

13. Saify K, Saadat M. Influence of a 50bp Ins/Del polymorphism at promoter of the superoxide dismutase-1 on gene expression and risk of heroin dependency. Environ Health Prev Med. 2017;22:4. doi:10.1186/s12199-0170617-8

14. Zendehboodi Z. Comparison of cytological parameters of exfoliated buccal mucosal cells in different temperament groups. J Kermanshah Univ Med Sci. 2017;21(1): 27-31.

15. Zendehboodi Z. Association of glutathione S-transferase $\mathrm{M} 1$ and $\mathrm{T} 1$ polymorphisms and temperament. Mol Biol Res Commun. 2017;6(3):95-100. doi: 10.22099/ mbrc.2017.4074

16. Mojahedi M, Naseri M, Majdzadeh R, et al. Reliability and validity assessment of mizaj questionnaire: a novel 
self-report scale in Iranian Traditional Medicine. Iran Red Crescent Med J. 2014;16(3):e15924. doi: 10.5812/ ircmj.15924

17. Dar FA, Zaidi IH, Sherani FS. Physiological variation of serum alkaline phosphatase level in damawi and balghami males in a sample population. Indian J Traditional Knowledge. 2011;10(4):741-744.

18. Shahabi S, Hassan Z, Mahdavi M, et al. Hot and cold natures and some parameters of neuroendocrine and immune systems in Traditional Iranian Medicine: a preliminary study. J Altern Complement Med. 2008;14(2):147-156. doi:10.1089/acm.2007.0693.

19. Ali SM, Islam R, Alam M. A scientific correlation between blood groups and temperaments in Unani medicine. Indian J Traditional Knowledge. 2007;6(2):319-323.

20. Mirtaheri E, Namazi N, Nafiseh Sargheini N, Heshmati J, Hadi V. Different types of Mizaj (temperament) in relation with body composition in overweight and obese women: Avicenna's opinion. Indian $\mathrm{J}$ Traditional Knowledge. 2015;14(2):240-243.

21. Ma T, Tan C, Zhang H, Wang M, Ding W, Li S. Bridging the gap between traditional Chinese medicine and systems biology: the connection of cold Syndrome and NEI network. Mol Biosyst. 2010;6(4):613-619. doi: 10.1039/ b914024g

22. Rezadoost H, Karimi M, Jafari M. Proteomics of hot-wet and cold-dry temperaments proposed in Iranian traditional medicine: a Network-based Study. Sci Rep. 2016;6:30133. doi:10.1038/srep30133

23. Chen G, Lu C, Zha Q, et al. A network-based analysis of traditional Chinese medicine cold and hot patterns in rheumatoid arthritis. Complement Ther Med. 2012;20(12):23-30. doi:10.1016/j.ctim.2011.10.005. 